\title{
A Noninvasive Score to Predict Liver Fibrosis in HBeAg-Positive Hepatitis B Patients with Normal or Minimally Elevated Alanine Aminotransferase Levels
}

\author{
Yanping Chen, ${ }^{1,2}$ Yanping $\mathrm{Li}^{2}{ }^{2} \mathrm{Na} \mathrm{Li},{ }^{1}$ Xiude Fan, ${ }^{1}$ Chunyan Li, ${ }^{1,2}$ Pingping Zhang, ${ }^{1,2}$ \\ Qunying Han $\mathbb{D D}^{1}$, and Zhengwen Liu $\mathbb{D}^{1}$ \\ ${ }^{1}$ Department of Infectious Diseases, First Affiliated Hospital of Xi'an Jiaotong University, Xi'an, 710061 Shaanxi, China \\ ${ }^{2}$ Department of Infectious Diseases, Affiliated Hospital of Yan'an University, Yan'an, 716000 Shaanxi, China \\ Correspondence should be addressed to Zhengwen Liu; liuzhengwen113@xjtu.edu.cn
}

Received 11 August 2018; Revised 1 September 2018; Accepted 9 September 2018; Published 14 October 2018

Academic Editor: Timo Sorsa

Copyright ( 2018 Yanping Chen et al. This is an open access article distributed under the Creative Commons Attribution License, which permits unrestricted use, distribution, and reproduction in any medium, provided the original work is properly cited.

\begin{abstract}
Noninvasive fibrosis tests are highly needed but have not been well studied in chronic hepatitis B patients with normal or minimally elevated alanine aminotransferase (ALT) levels. This study is aimed at developing a noninvasive score system to predict liver fibrosis in these patients. HBeAg-positive chronic hepatitis B patients with ALT levels of $<80 \mathrm{IU} / \mathrm{l}$ and liver histology $(n=290)$ were assigned to training $(n=203)$ or validation $(n=87)$ groups. Training group patients were divided into nonsignificant (F01) and significant fibrosis (F2-4) according to METAVIR stages. Logistic regression was performed to identify factors for liver fibrosis and develop a score system. The capacity of the score to identify the severity of fibrosis was displayed by receiver operating characteristic curve (ROC) and area under ROC (AUROC) values. Multivariate logistic regression showed that $\mathrm{HBeAg}$ (ratios of the sample to the cutoff values $(\mathrm{S} / \mathrm{CO})$ ) and liver stiffness measurement (LSM; kilopascals $(\mathrm{kPa})$ ) were independent factors of liver fibrosis. A score system composed of HBeAg and LSM by assigning a point of 1,2, or 3 to different HBeAg and LSM levels, respectively, was developed. The scores 2-3, 4, and 5-6 of the sum of HBeAg and LSM points indicated nonsignificant, indeterminate, and significant fibrosis, respectively. The score system had an AUROC of 0.880 and showed similar performance in validation group patients. The accuracy for identifying significant and nonsignificant fibrosis was $77.14 \%$ in validation group patients and $71.26 \%$ in the entire group of patients. It is suggested that this noninvasive score system can accurately predict hepatic fibrosis and may reduce the need for liver biopsy in HBeAg-positive patients with normal or minimally elevated ALT levels.
\end{abstract}

\section{Introduction}

Chronic hepatitis B virus (HBV) infection may induce chronic hepatitis, liver cirrhosis, and hepatocellular carcinoma (HCC) and affects nearly 250 million people worldwide [1]. The course of chronic HBV infection is a dynamic process in which the individuals with HBV infection may experience different clinical phases displaying variable levels of serum alanine aminotransferase (ALT), HBV DNA, and HBV antigens [2]. The levels of serum ALT and HBV DNA and the severity of hepatic fibrosis are important predictors of long-term outcome and indicators of treatment initiation and response assessment $[2,3]$.
The indication of fibrosis or inflammation in patients with highly elevated ALT levels or cirrhosis is relatively easier. However, in HBV-infected patients with persistent borderline normal or slightly elevated ALT levels, liver biopsy is recommended [2] since it remains the gold standard for the evaluation of liver fibrosis and inflammation in chronic liver disease [4]. Liver biopsy is an invasive examination with high cost and certain pitfalls such as discomfort in patients, association of complications, and sampling errors $[5,6]$. It is particularly difficult for patients to undergo multiple liver biopsies in order to serially monitor disease progression or to guide treatment. As a result, noninvasive approaches using readily available and noninvasive parameters have been 
developed for assessing the extent of liver fibrosis [7-13]. However, most of these methods were developed and used for hepatic fibrosis in hepatitis $\mathrm{C}$ virus (HCV) infection and/or HCV/human immunodeficiency virus (HIV) coinfection. Validation and comparisons of these models for liver fibrosis in chronic HBV infection showed unideal or inconsistent findings or diminished accuracy [14-22].

Recently, a model, gamma-glutamyltranspeptidase(GGT-) to-platelet ratio (GPR), was developed to predict significant liver fibrosis and cirrhosis in patients with chronic HBV infection [23]. Some studies indicated that GPR had better performance in comparison with aspartate aminotransferase- (AST-) to-platelet ratio index (APRI) and fibrosis index based on 4 factors (FIB-4) in predicting liver fibrosis of chronic hepatitis B [24] and in improving the sensitivity and specificity of hepatic fibrosis assessment in chronic hepatitis B when combined with APRI or FIB-4 [25]. However, a study comparing seventeen noninvasive models including GPR showed that the models evaluated were not appropriate for all situations of chronic HBV infection [21]. Other studies showed that GPR does not add any advantage over APRI and FIB-4 in identifying significant fibrosis, severe fibrosis, and cirrhosis in chronic hepatitis B patients [26-28].

Few studies have been conducted to develop or evaluate noninvasive approaches in identifying liver fibrosis in hepatitis B e-antigen- ( $\mathrm{HBeAg-}$ ) positive chronic hepatitis B patients with normal or minimally elevated ALT levels (usually ALT of $<2$ upper limit of normal value (ULN)). APRI and FIB-4 were not indicated to be ideal hepatic fibrosis markers in these patients [20]. A model, globulin-platelet model (GP), was shown to be a more accurate noninvasive fibrosis model than APRI and FIB-4 to diagnose significant fibrosis and cirrhosis in chronic hepatitis B patients with high HBV DNA and mildly elevated ALT levels [29], but it has not been validated. Notably, liver stiffness measurement (LSM) by transient elastography, a noninvasive technique, was shown to be superior to APRI and FIB-4 for indicating liver fibrosis in $\mathrm{HBV}$-infected patients with persistently normal ALT levels [20]. LSM was also shown to be a reliable noninvasive examination for the diagnosis of liver fibrosis in $\mathrm{HBeAg}$-positive patients with high HBV DNA and normal or mildly elevated ALT levels [30]. However, the value of other noninvasive demographic, clinical, and laboratory parameters, especially their combination with LSM, needs to be further clarified in identifying liver fibrosis in $\mathrm{HBeAg}-$ positive patients with ALT of $<2 \times$ ULN. Therefore, this study is aimed at developing a noninvasive approach by including LSM and routinely available noninvasive parameters in the analysis for the evaluation of liver fibrosis in $\mathrm{HBeAg-}$ positive patients with ALT of $<2 \times$ ULN based on the fibrosis staging by liver biopsy. The results were also compared with the most discussed models, APRI and FIB-4, and the newly developed GPR.

\section{Materials and Methods}

2.1. Patients. The data of $290 \mathrm{HBeAg-positive} \mathrm{chronic} \mathrm{hepa-}$ titis B patients undergoing liver biopsy were collected from the Affiliated Hospital of Yan'an University from October
2013 to February 2018. Chronic HBV infection was defined as the persistent positivity of hepatitis B surface antigen (HBsAg), HBeAg, and antibody to hepatitis B core antigen (anti-HBc) for more than 6 months [31].

Inclusion criteria of patients were age $\geq 18$ years; $\mathrm{HBsAg}$, $\mathrm{HBeAg}$, and HBV DNA positive for more than 6 months; serum ALT of $<2 \times$ ULN; and signed informed consent for liver biopsy. Exclusion criteria of patients were: coinfection with other hepatotropic viruses such as HCV, hepatitis A virus, hepatitis $\mathrm{E}$ virus, or other viruses such as HIV; existence of other liver diseases including alcoholic, nonalcoholic, drug-induced, autoimmune liver diseases, and decompensated liver cirrhosis or tumors; diseases causing extrahepatic organ fibrosis; a history of antiviral therapy (interferon or nucleos(t)ides); pregnancy; and use of anticoagulant 1 week before liver biopsy. This study was performed to conform to the Declaration of Helsinki and was approved by the Ethics Committee of the Affiliated Hospital of Yan'an University (No. 2016-30).

2.2. Laboratory Tests. Fasting vein blood was collected for laboratory tests within 1 week of liver biopsy. Hyaluronic acid (HA), laminin, type III procollagen (PCIII), and type IV collagen (IV-C) were determined using Autodesk Chemiluminescent Immunoassay Analyzer (Yantai, China) and reagents from Beijing Yuande Bio-Medical Engineering Co., Ltd. (Beijing, China). HBV DNA quantification was performed using Abbott RealTime HBV assay with the Abbott m2000 SystemDNA reagents (Abbott Molecular Inc., Des Plaines, IL, USA) and ABI7500 Quantitative Cycler (ABI, USA). HBsAg was quantitatively determined by chemiluminescent microparticle immunoassay (CMIA) of ARCHITECT HBsAg assay (Abbott Ireland, Sligo, Ireland). HBeAg and anti-HBc were determined by CMIA of ARCHITECT HBeAg assay (Abbott $\mathrm{GmbH} \&$ Co. KG, Wiesbaden, Germany) and anti-HBc II assay (Abbott GmbH \& Co. KG, Wiesbaden, Germany), respectively, and the ratios of the sample to the cutoff values (S/CO) were used as HBeAg and anti-HBc levels, respectively.

\subsection{Imaging Examination and Liver Stiffness Measurement.} The thickness of the spleen and the inner diameter of the portal vein were measured using a Toshiba Ultrasound (Toshiba, Japan). LSM (kilopascals $(\mathrm{kPa})$ ) was performed using a liver transient elastography FibroScan System (Echosens SAS, Paris, France). The operator was blinded to the clinical characteristics of the patients. The median value of LSM was used to represent liver stiffness.

2.4. Liver Biopsy and Pathological Diagnosis. Percutaneous liver biopsy was performed with local anesthesia under the guidance of ultrasonography. Liver samples of about 1.5$3 \mathrm{~cm}$ in length were fixed with $10 \%$ formaldehyde solution and embedded with paraffin for histological analysis. Liver histology was interpreted by two hepatology pathologists who were blinded to the clinical information and the results of noninvasive tests. According to the METAVIR scoring system [32], liver fibrosis was classified into five stages: F0, no fibrosis; F1, portal fibrosis without septa; F2, portal 
fibrosis with rare septa; F3, numerous septa without cirrhosis; and F4, cirrhosis.

2.5. Grouping of Training and Validation Patients. The study population was divided into a training group and a validation group according to the recruiting time. Patients recruited from October 2013 to October 2016 were included in the training group $(n=203)$ and patients recruited from November 2016 to February $2018(n=87)$ were included in the validation group. According to the METAVIR scoring system [32], liver fibrosis was classified as nonsignificant (F0-1) and significant fibrosis (F2-4).

2.6. Statistical Analysis. SPSS version 20.0 software (SPSS Inc., Chicago, IL, USA) was used for statistical analysis. Data with normal distribution were expressed as the mean \pm standard deviation (SD), and comparison was performed by $t$ test. Data with nonnormal distribution was expressed as median and interquartile range (median (IQR)). Comparisons were performed using a nonparametric test (MannWhitney $U$ test). The dichotomous data were expressed by composition ratio. The comparison was carried out by $\chi^{2}$ test. Liver pathological examination of fibrosis was used as the gold standard, and risk factors for liver fibrosis were analysed by logistic regression. A score system was derived from the independent factors. The diagnostic ability of the score system for liver fibrosis was evaluated using the receiver operating characteristic curve (ROC). The cutoff values were determined by the ROC at maximum Youden's index with optimal sensitivity and specificity. The area under the ROC (AUROC) was compared using MedCalc Software. A twotailed value of $p<0.05$ indicated a statistical significance.

\section{Results}

3.1. Patient Characteristics. Of the $290 \mathrm{HBeAg-positive}$ chronic hepatitis B patients, 218 patients (75.2\%) had nonsignificant hepatic fibrosis (F0-1) and 72 patients $(24.8 \%)$ had significant hepatic fibrosis (F2-4) according to the liver pathological fibrosis stage.

The characteristics of patients in the training and validation groups and the differences between the two groups are shown in Table 1. Of note, the training group had a higher percent of patients with fibrosis F0-1 (80.79\%, F0-1/ $\mathrm{F} 2-4=164 / 39)$ than the validation group $(62.07 \%, \mathrm{~F} 0-1 /$ F2-4 $=54 / 33, p=0.001$, Table 1 ).

3.2. Noninvasive Parameters Associated with Hepatic Fibrosis in the Training Group. The fibrosis stages in the 203 training group patients were as follows: F0, 13 patients (6.4\%); F1, 151 patients (74.4\%); F2, 28 patients (13.8\%); F3, 9 patients (4.4\%); and F4, 2 patients (1.0\%). Scilicet, $164(80.8 \%)$ patients, had nonsignificant liver fibrosis (F0-1 group) and 39 patients (19.2\%) had significant hepatic fibrosis (F2-4). The comparison of parameters between the patients with F0-1 and those with F2-4 fibrosis are shown in Supplementary Table 1. Compared with nonsignificant fibrosis patients (F0-1), patients with significant liver fibrosis (F24) had lower HBV DNA, HBsAg, HBeAg, and platelet count levels and higher anti-HBc, alpha-fetoprotein (AFP), AST, globulin, GGT, laminin, IV-C, LSM, and spleen thickness (Supplementary Table 1).

Multivariate logistic regression showed that lower HBeAg level (OR: 0.391, 95\% CI: 0.241-0.632, $p<0.001$ ) and higher LSM (OR: 1.522, 95\% CI: 1.274-1.819, $p<$ 0.001 ) were independent predictors of significant liver fibrosis (Table 2).

\subsection{Development of a Score System and Performance in the} Training Group. Based on multivariate logistic regression, a formula, $P=e^{\text {logistic }} /\left(1+e^{\text {logistic }}\right)$, was derived, where logi stic $=-1.988-0.94 \mathrm{Lg} \mathrm{HBeAg}+0.42 \mathrm{LSM}$. By compound function derivation and operation $[33,34]$, the formula was optimized as follows: $P=1.6375-0.4326 \mathrm{HBeAg}+0.4451 \mathrm{~L}$ SM. Because the coefficients of the variables HBeAg and LSM were almost equal and their ratio approximated 1, the formula was simplified as $P=-\mathrm{HBeAg}+\mathrm{LSM}$ to facilitate calculation.

The lower and upper cutoff values for $\mathrm{HBeAg}$ were 1247.38 S/CO and 106.91 S/CO, respectively, and the values for LSM were $4.95 \mathrm{kPa}$ and $8.50 \mathrm{kPa}$, respectively (Table 3). To develop a score system, HBeAg and LSM were, respectively, assigned to different points from 1 to 3 according to the cutoff values, and the sum of HBeAg and LSM points (score) was used for staging liver fibrosis (Table 4). The scores 3 and 5 corresponding to a sensitivity of $92.3 \%$ and a specificity of $98.8 \%$ were determined to be the cutoff values of the score system, namely, scores 2-3, 4, and 5-6 indicated nonsignificant hepatic fibrosis, indeterminate fibrosis, and significant fibrosis, respectively (Table 4). The 203 patients in the training group were scored according to this system, and the AUROC was 0.880 (95\% CI: 0.827, 0.921) for differentiating significant and nonsignificant fibrosis $(p<0.001$, Supplementary Figure 1).

According to the pathological diagnosis of the liver tissue, the score for classifying hepatic fibrosis had a sensitivity of $90.0 \%$ and a specificity of $85.7 \%$. The accuracy was $86.5 \%$, positive predictive value was $58.7 \%$, and negative predictive value was $97.4 \%$. The positive likelihood ratio and negative likelihood ratio were 6.30 and 0.12 , respectively.

The AUROC of the score (0.880) was significantly higher than HBeAg (0.822), LSM (0.791), APRI (0.720), FIB-4 (0.671), and GPR $(p=0.023, p=0.0079, p=0.0002, p<$ 0.0001 , and $p<0.0001$, respectively, Table 5, Figure 1).

3.4. Validation of the Score System. The fibrosis stages in the 87 validation group patients were as follows: F0, 11 patients (12.6\%); F1, 43 patients (49.4\%); F2, 23 patients (26.4\%); F3, 9 patients $(10.3 \%)$, and $F 4,1$ patient (1.1\%), in which 54 patients $(62.1 \%)$ had nonsignificant fibrosis (F0-1) and 33 patients $(37.9 \%)$ had significant fibrosis (F2-4).

The AUROC of the score for predicting liver fibrosis in this group patients was $0.727(0.612,0.842)$. Because there were differences between the validation group and the training group patients in the fibrosis stages and the validation group had a relatively small sample size (Table 1), the AUROC was standardized by the methods as described elsewhere [35]. The adjusted AUROC was calculated to be 0.835 (95\% CI: 0.612, 0.842), which had no significant difference 
TABLE 1: Characteristics of patients in the training and validation groups.

\begin{tabular}{|c|c|c|c|}
\hline & Training group & Validation group & $p$ \\
\hline$N$ & 203 & 87 & \\
\hline Age (years) & $31(26,41)$ & $34(29,40)$ & 0.067 \\
\hline Gender (male/female) & $121 / 82$ & $50 / 37$ & 0.735 \\
\hline BMI $\left(\mathrm{kg} / \mathrm{m}^{2}\right)$ & $23.234 \pm 3.337$ & $23.550 \pm 2.964$ & 0.447 \\
\hline Family member of HBV infection (yes/no) & $143 / 60$ & $61 / 26$ & 0.955 \\
\hline Smoking (yes/no) & $41 / 162$ & $24 / 63$ & 0.167 \\
\hline Drinking (yes/no) & $26 / 177$ & $9 / 78$ & 0.555 \\
\hline Lg HBV DNA (IU/ml) & $7.544(6.839,7.968)$ & $8.358(6.340,8.727)$ & $<0.001$ \\
\hline Lg HBsAg (IU/ml) & $4.411(3.811,4.751)$ & $4.424(3.636,4.834)$ & 0.575 \\
\hline Lg HBeAg (S/CO) & $3.098(2.442,3.162)$ & $3.139(2.217,3.195)$ & 0.103 \\
\hline Anti-HBc (S/CO) & $9.800(8.300,11.000)$ & $8.840(7.440,9.520)$ & $<0.001$ \\
\hline WBC $\left(\times 10^{9} / 1\right)$ & $5.700(4.700,6.510)$ & $5.830(4.920,7.030)$ & 0.219 \\
\hline $\operatorname{RBC}\left(\times 10^{12} / \mathrm{l}\right)$ & $3.357 \pm 1.246$ & $4.904 \pm 0.611$ & $<0.001$ \\
\hline Platelet $\left(\times 10^{9} / \mathrm{l}\right)$ & $198.515 \pm 51.570$ & $194.690 \pm 58.548$ & 0.579 \\
\hline AFP (ng/ml) & $5.200(3.400,9.200)$ & $2.670(1.780,4.140)$ & $<0.001$ \\
\hline ALT (IU/l) & $32.000(25.000,46.000)$ & $32.000(21.000,51.000)$ & 0.661 \\
\hline AST (IU/l) & $26.000(20.000,33.000)$ & $28.000(21.000,35.000)$ & 0.146 \\
\hline Tbil $(\mu \mathrm{mol} / \mathrm{l})$ & $11.000(8.000,15.000)$ & $11.100(7.900,13.700)$ & 0.526 \\
\hline $\operatorname{Dbil}(\mu \mathrm{mol} / \mathrm{l})$ & $4.000(3.000,5.500)$ & $4.400(3.300,5.800)$ & 0.112 \\
\hline Albumin (g/l) & $42.231 \pm 4.617$ & $40.872 \pm 4.418$ & 0.021 \\
\hline Globulin (g/l) & $28.312 \pm 5.293$ & $28.339 \pm 4.433$ & 0.967 \\
\hline GGT (IU/l) & $18.000(12.000,30.000)$ & $18.000(11.000,25.000)$ & 0.674 \\
\hline INR & $1.052 \pm 0.059$ & $0.956 \pm 0.060$ & $<0.001$ \\
\hline $\mathrm{HA}(\mathrm{ng} / \mathrm{ml})$ & $42.930(17.000,71.000)$ & $50.290(37.950,62.220)$ & 0.090 \\
\hline Laminin (ng/ml) & $29.000(8.000,63.000)$ & $50.040(29.460,72.130)$ & $<0.001$ \\
\hline IV-C (ng/ml) & $34.000(15.100,58.000)$ & $43.220(23.420,67.280)$ & 0.014 \\
\hline PC-III (ng/ml) & $2.000(0.200,4.000)$ & $4.410(3.320,6.100)$ & $<0.001$ \\
\hline LSM (kPa) & $5.400(4.700,6.500)$ & $4.900(4.200,6.600)$ & 0.063 \\
\hline Portal vein width $(\mathrm{cm})$ & $1.100(1.110,1.200)$ & $1.200(1.100,1.200)$ & 0.001 \\
\hline Spleen thickness $(\mathrm{cm})$ & $3.323 \pm 0.593$ & $3.393 \pm 0.446$ & 0.330 \\
\hline Liver fibrosis staging (F0-1/F2-4) & $164 / 39$ & $54 / 33$ & 0.001 \\
\hline
\end{tabular}

BMI: body mass index; WBC: white blood cell; RBC: red blood cell; AFP: alpha-fetoprotein; ALT: alanine aminotransferase; AST: aspartate aminotransferase; Tbil: total bilirubin; Dbil: direct bilirubin; GGT: gamma-glutamyltranspeptidase; INR: international normalized ratio; HA: hyaluronic acid; IV-C: type IV collagen; PC-III: type III procollagen; LSM: liver stiffness measurement.

TAвLE 2: Results of logistic regression analysis of independent factors associated with liver fibrosis in the training group patients.

\begin{tabular}{|c|c|c|c|c|c|c|c|}
\hline \multirow{2}{*}{ Variable } & \multirow{2}{*}{$B$} & \multirow{2}{*}{ SE } & \multirow{2}{*}{ Wals } & \multirow{2}{*}{$\operatorname{Exp}(B)$} & \multicolumn{2}{|c|}{$95 \%$ CI of $\exp (B)$} & \multirow[b]{2}{*}{$p$} \\
\hline & & & & & Lower & Upper & \\
\hline Constant & -1.988 & 0.910 & 4.767 & 0.137 & - & - & 0.029 \\
\hline Lg HBeAg & -0.940 & 0.246 & 14.649 & 0.391 & 0.241 & 0.632 & $<0.001$ \\
\hline LSM & 0.420 & 0.091 & 21.417 & 1.522 & 1.274 & 1.819 & $<0.001$ \\
\hline
\end{tabular}

$B$ : independent variable coefficient; SE: standard error; LSM: liver stiffness measurement; Lg HBeAg: HBeAg after log10 transformation.

compared with the training group (0.880, 95\% CI: 0.827 , $0.921 ; z=0.677, p>0.05$, Supplementary Table 2).

Of the 87 patients in the validation group, 53 patients were predicted to have scores 2 and 3, 17 patients have score 4 , and 17 patients have scores 5 and 6 , respectively, according to the score system. Of the patients with scores
$2-3,41 / 53$ (77.36\%) had nonsignificant fibrosis and 13/17 (76.47\%) patients with scores 5-6 had significant fibrosis. The total diagnostic accuracy of scores 2-3 and scores 5-6 was $77.14 \%$. In the 17 patients with score 4,9 cases had nonsignificant fibrosis and 8 cases had significant fibrosis. In the validation group, $62.07 \%$ of the patients 
TABLE 3: Cutoff values of HBeAg and LSM for classifying liver fibrosis.

\begin{tabular}{lcccccc}
\hline Exploratory factor & Sensitivity (\%) & Youden's index & Specificity (\%) & Youden's index & Lower cutoff & Upper cut-off \\
\hline HBeAg & 92.3 & 0.527 & 90.2 & 0.440 & $1247.38^{*}$ & $106.91^{*}$ \\
LSM & 92.3 & 0.338 & 93.3 & 0.497 & $4.95^{* *}$ & $8.50^{* *}$ \\
\hline
\end{tabular}

LSM: liver stiffness measurement. ${ }^{*}$ Values in S/CO; ${ }^{* *}$ values in $\mathrm{kPa}$.

TABle 4: Point assignment of the score system for noninvasive diagnosis of liver fibrosis.

\begin{tabular}{lccc}
\hline Exploratory factor & 1 point & 2 points & 3 points \\
\hline HBeAg $(\mathrm{S} / \mathrm{CO})$ & $>1248$ & $106-1248$ & $<106$ \\
LSM $(\mathrm{kPa})$ & $<4.9$ & $4.9-8.5$ & $>8.5$ \\
\hline
\end{tabular}

LSM: liver stiffness measurement. Score $=$ the point of $\mathrm{HBeAg}+$ the point of LSM. Score 2-3: nonsignificant fibrosis; score 4: indeterminate fibrosis; and score 5-6: significant fibrosis.

TABLE 5: Comparison of performance of the score system with HBeAg, LSM, APRI, FIB-4, and GPR for liver fibrosis.

\begin{tabular}{lcccc}
\hline & AUROC $(95 \% \mathrm{CI})$ & SE & $Z$ & $p^{*}$ \\
\hline Score system & $0.880(0.827,0.921)$ & 0.0304 & & \\
HBeAg & $0.822(0.762,0.872)$ & 0.0365 & 2.273 & 0.023 \\
LSM & $0.791(0.728,0.845)$ & 0.0443 & 2.654 & 0.0079 \\
APRI & $0.720(0.653,0.780)$ & 0.0461 & 3.784 & 0.0002 \\
FIB-4 & $0.671(0.601,0.735)$ & 0.0502 & 4.247 & $<0.0001$ \\
GPR & $0.687(0.619,0.750)$ & 0.0489 & 4.190 & $<0.0001$
\end{tabular}

${ }^{*}$ Compared with the score system. LSM: liver stiffness measurement; APRI: aspartate aminotransferase-to-platelet ratio index; FIB-4: fibrosis index based on 4 factors; GPR: gamma-glutamyltranspeptidase-to-platelet ratio.

can be accurately predicted for the degree of hepatic fibrosis by the score system.

\section{Discussion}

Previous studies showed that in HBeAg-positive patients with ALT of $\leq 2 \times$ ULN, the presence of significant liver fibrosis $(\geq F 2)$ was $30.2 \%$ [36]. In HBeAg-positive patients with persistently normal ALT and ALT 1-2× ULN, significant fibrosis was found in $49.4 \%$ and $69.8 \%$ of the patients, respectively [37]. In HBeAg-positive patients with persistently normal or intermittently elevated ALT, histologic fibrosis stage of $\geq 2$ was found in $40.2 \%$ and $65.5 \%$ of the patients, respectively [38]. In the present study, $24.8 \%$ (72/218) of the patients with ALT of $<2 \times$ ULN had significant hepatic fibrosis (F2-4). Although there are differences in the proportion of liver fibrosis stages between the studies which may be related to the age and the definition of ALT levels, it is clearly revealed that nearly more than $25 \%$ of HBeAg-positive patients with ALT of $<80$ IU/l had significant liver fibrosis.

Many factors have been examined for the role in evaluating liver fibrosis in chronic hepatitis B patients. In our study, various demographic, clinical, and laboratory parameters were included in the analysis in relation to liver fibrosis in HBeAg-positive chronic hepatitis B patients with ALT of

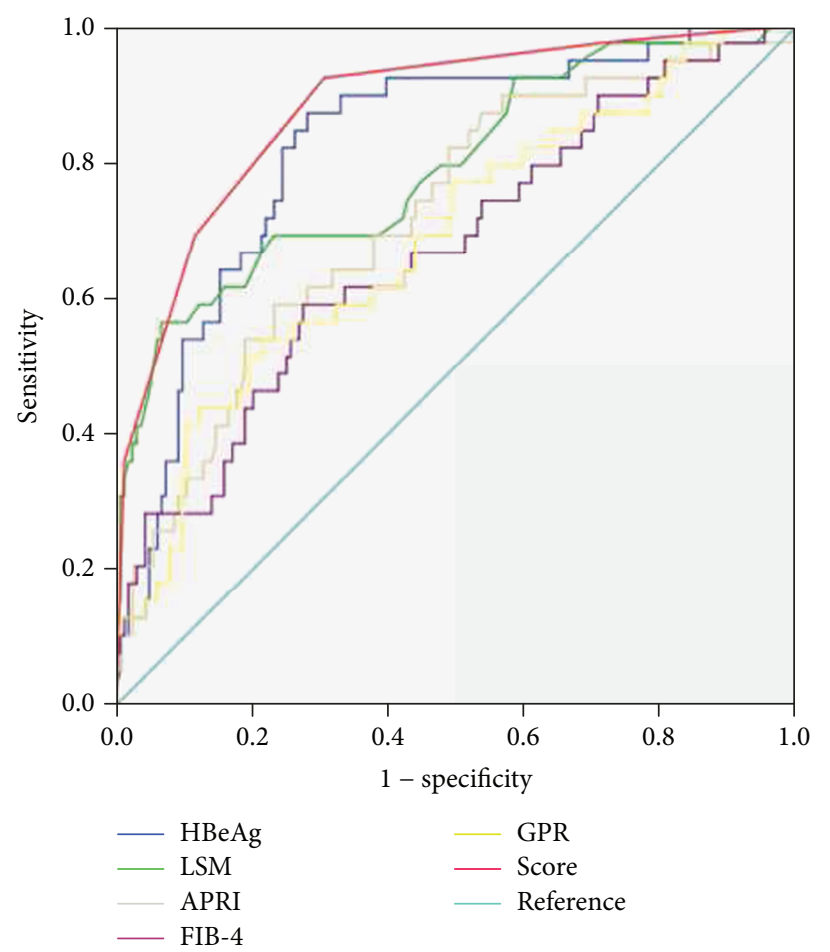

FIGURE 1: Comparison of the receiver operating characteristic curves of the score with HBeAg, LSM, aspartate aminotransferase(AST-) to-platelet ratio index (APRI), fibrosis index based on 4 factors (FIB-4), and gamma-glutamyltranspeptidase-to-platelet ratio (GPR) for predicting significant from nonsignificant liver fibrosis in the training group patients.

$<2 \times$ ULN. Patients with significant liver fibrosis (F2-4), in comparison with nonsignificant fibrosis (F0-1), had lower HBV DNA, HBsAg, HBeAg, and platelet count levels and higher anti-HBc, AFP, AST, globulin, GGT, laminin, IV-C, LSM, and spleen thickness. These results are mostly consistent with previous studies showing that lower serum HBsAg, HBV DNA, and platelet count [39-42] and higher AFP, AST, globulin, GGT, laminin, and IV-C levels [29, 42-45] are associated with significant fibrosis in chronic hepatitis B patients. Older age was suggested to be related to significant fibrosis in chronic hepatitis B patients in previous studies $[39,41,42,46$, 47]. However, our study did not show a relationship between patient age and liver fibrosis. This may be related to the patient population included in our study because $\mathrm{HBeAg}$ positive chronic hepatitis B patients with ALT of $<2 \times$ ULN are usually younger and the age range of the patients has small deviations. Higher ALT levels were also indicated to be associated with increased risk of advanced liver fibrosis in some previous studies [47, 48]. In the present study, ALT levels were not indicated to be a factor associated with liver 
fibrosis. The inclusion of patients with ALT of $<2 \times$ ULN in the study may be partly related to this result because the magnitude of ALT fluctuation is small in this subgroup of patients. A previous study also showed that an elevated ALT level was not predictive of significant fibrosis for $\mathrm{HBeAg}$-positive disease [41]. Moreover, the degree of liver fibrosis in chronic HBV infection is determined by complex interaction of multiple factors. A recent study showed that, in patients with ALT of $>20$ but $\leq 40 \mathrm{IU} / 1$, age, ALT, and GGT were independent predictors of significant liver histological changes including significant fibrosis, while in patients with ALT of $\leq 20 \mathrm{IU} / \mathrm{l}$, age was the only independent predictor of significant liver histological changes [46]. Albumin levels had no significant difference between patients with significant and nonsignificant fibrosis in $\mathrm{HBeAg}$-positive chronic hepatitis $B$ patients with ALT of $<2 \times$ ULN in the present study. This is consistent with most of previous investigations. Anti-HBc and spleen thickness were rarely evaluated previously, and higher level of anti-HBc and wider thickness of spleen were observed in patients with significant hepatic fibrosis in the present study. Further studies are required to confirm the role of anti-HBc and spleen thickness in assessing liver fibrosis in chronic HBV infection.

Multiple logistic regression analysis using liver pathological examination of fibrosis as the gold standard showed that HBeAg levels and LSM are independent predictors of the hepatic fibrosis, and a novel score system composed of HBeAg and LSM levels was derived in the present study. HBeAg quantitation has been shown to have a moderate predictive value for discriminating immune tolerant phase and immune clearance phase in chronic $\mathrm{HBV}$ infection [49]. Serum HBeAg levels were indicated to be negatively correlated with the severity of liver inflammation in HBeAg-positive chronic hepatitis B patients [50], and high serum $\mathrm{HBeAg}$ levels were suggested to accurately predict insignificant histology among $\mathrm{HBeAg}$-positive patients with ALT of $<2 \times$ ULN [51]. HBeAg has been found to induce the activation and proliferation of hepatic stellate cells [52] which are pivotal players in the development of hepatic fibrosis. Consistently, HBeAg levels showed a good predictive value for liver fibrosis in $\mathrm{HBeAg}$-positive patients with ALT of $<2 \times$ ULN in the present study. It is suggested that $\mathrm{HBeAg}$ is involved in the pathology of liver fibrosis in chronic HBV infection and its levels may indicate the degree of liver fibrosis.

LSM by transient elastography is widely used for the noninvasive evaluation of liver fibrosis. It is indicated to be a reliable noninvasive test for the diagnosis of liver fibrosis in chronic hepatitis B patients with ALT of $\leq 2 \times$ ULN [30]. It is superior to current serobiomarkers in chronic hepatitis $B$ patients with various levels of ALT $[53,54]$. It is also superior to APRI and FIB-4 in chronic hepatitis B patients with persistently normal ALT levels [20]. Moreover, LSM is superior to FibroTest in the noninvasive identification of fibrosis among HCV carriers with normal/near-normal aminotransferases [55]. LSM displayed a good predictive value for liver fibrosis in $\mathrm{HBeAg}$-positive patients with ALT of $<2 \times$ ULN in the present study. Of note, enhanced inflammatory activity as indicated by elevated ALT can lead to elevated LSM values unrelated to histological fibrosis stage and can result in the overestimation of fibrosis $[20,53]$. Therefore, LSM appears to have an advantage in classifying liver fibrosis in hepatitis $B$ patients with ALT of $<2 \times$ ULN because of the avoidance of potential influence by significantly elevated ALT levels.

The inclusion of parameters that directly reflect $\mathrm{HBV}$ replication $(\mathrm{HBeAg})$ and reflect liver fibrosis (LSM) can better reflect the involvement of factors associated with liver fibrosis in HBeAg-positive patients with normal or slightly elevated ALT levels. In fact, the score system developed in this study is superior to the most used models APRI and FIB-4 and the newly developed GPR for predicting liver fibrosis in HBeAg-positive chronic hepatitis $\mathrm{B}$ patients with ALT of $<2 \times$ ULN.

The score system was validated in the validation group patients with similar performance as in the training group patients. In addition to high sensitivity and specificity with high diagnostic accuracy and reproducibility, the score system is easy to calculate and simple to use and has better patient acceptance and higher speed of result obtainability with the noninvasive nature. This score may be especially useful for dynamic evaluation of liver fibrosis and may reduce the need for liver biopsy, making clinical care safer and more convenient for HBeAg-positive patients with ALT of $<2 \times$ ULN.

Although the score system developed has advantages in evaluating liver fibrosis in this particular subgroup of patients, it is not perfect, in that the system has not been validated in large patient population with various demographic, clinical, and laboratory variations. For example, the patients included in our study had normal body mass index (BMI) while technical failure of LSM was shown to be common in patients with $\mathrm{BMI} \geq 28 \mathrm{~kg} / \mathrm{m}^{2}$ [56]. Therefore, additional studies evaluating the diagnostic accuracy of the score system are needed. In addition, the model may currently be unable to be adopted in some clinical practice settings, but it indicates an important direction toward more accurate evaluation of liver fibrosis with the increasing application of $\mathrm{HBeAg}$ determination and LSM usage.

In summary, the novel score system composed of noninvasive parameters, HBeAg and LSM, can accurately differentiate hepatic fibrosis and may reduce the need for liver biopsy in these subgroups of patients. Additional studies are needed to confirm the diagnostic accuracy of the score system and to evaluate the usefulness for identifying patients with significant fibrosis who might benefit from antiviral therapy.

\section{Data Availability}

The data underlying the findings of this study are all included in the manuscript and in the Supplementary Materials section of this manuscript.

\section{Disclosure}

The funders have no role in the study design, data collection and analysis, decision to publish, or preparation of the manuscript. 


\section{Conflicts of Interest}

The authors declare that they have no conflicts of interest related to this paper or the study it describes.

\section{Acknowledgments}

We thank Professor Guanghua Xu, Dr. Tai Wang, Dr. Haimei Wang, Dr. Ruiqin Zhang, and Dr. Yuan Li from the Department of Infectious Diseases, Affiliated Hospital of Yan'an University and Dr. Yan Li, Dr. Xiaoyan Zeng, Dr. Fan Gao, Dr. Yawen Wang, Dr. Guoyu Zhang, Dr. Man Li, and Dr. Zhu Li from the First Affiliated Hospital of Xi'an Jiaotong University for their help during this study. This study was supported by Key R\&D Project of China Shaanxi Provincial Department of Science and Technology (2017 SF-269), Special Scientific Research Project of China, Education Department of Shaanxi Province (17JK0866), and National Natural Science Foundation of China (Grant number: 81371798).

\section{Supplementary Materials}

Supplementary 1. Table 1: comparison of patients with different liver fibrosis stages (F0-1 vs. F2-4) in the training group.

Supplementary 2. Table 2: comparison of AUC values of the score system for classifying liver fibrosis in training and validation group patients.

Supplementary 3. Figure 1: receiver operating characteristic curve of the score for identifying significant from insignificant liver fibrosis in the training group patients.

\section{References}

[1] A. Schweitzer, J. Horn, R. T. Mikolajczyk, G. Krause, and J. J. Ott, "Estimations of worldwide prevalence of chronic hepatitis B virus infection: a systematic review of data published between 1965 and 2013," The Lancet, vol. 386, no. 10003, pp. 1546-1555, 2015.

[2] N. A. Terrault, A. S. F. Lok, B. J. McMahon et al., "Update on prevention, diagnosis, and treatment of chronic hepatitis $\mathrm{B}$ : AASLD 2018 hepatitis B guidance," Hepatology, vol. 67, no. 4, pp. 1560-1599, 2018.

[3] P. Lampertico, K. Agarwal, T. Berg et al., "EASL 2017 clinical practice guidelines on the management of hepatitis B virus infection," Journal of Hepatology, vol. 67, no. 2, pp. 370-398, 2017.

[4] A. A. Bravo, S. G. Sheth, and S. Chopra, "Liver biopsy," New England Journal of Medicine, vol. 344, no. 7, pp. 495-500, 2001.

[5] J. Perrault, D. B. McGill, B. J. Ott, and W. F. Taylor, "Liver biopsy: complications in 1000 inpatients and outpatients," Gastroenterology, vol. 74, no. 1, pp. 103-106, 1978.

[6] G. Colloredo, M. Guido, A. Sonzogni, and G. Leandro, "Impact of liver biopsy size on histological evaluation of chronic viral hepatitis: the smaller the sample, the milder the disease," Journal of Hepatology, vol. 39, no. 2, pp. 239-244, 2003.

[7] F. Imbert-Bismut, V. Ratziu, L. Pieroni, F. Charlotte, Y. Benhamou, and T. Poynard, "Biochemical markers of liver fibrosis in patients with hepatitis $\mathrm{C}$ virus infection: a prospective study," The Lancet, vol. 357, no. 9262, pp. 1069-1075, 2001.

[8] X. Forns, S. Ampurdanès, J. M. Llovet et al., "Identification of chronic hepatitis $\mathrm{C}$ patients without hepatic fibrosis by a simple predictive model," Hepatology, vol. 36, no. 4, pp. 986-992, 2002.

[9] C. T. Wai, J. K. Greenson, R. J. Fontana et al., "A simple noninvasive index can predict both significant fibrosis and cirrhosis in patients with chronic hepatitis C," Hepatology, vol. 38, no. 2, pp. 518-526, 2003.

[10] A. Sud, J. M. Hui, G. C. Farrell et al., "Improved prediction of fibrosis in chronic hepatitis $\mathrm{C}$ using measures of insulin resistance in a probability index," Hepatology, vol. 39, no. 5, pp. 1239-1247, 2004.

[11] A. S. F. Lok, M. G. Ghany, Z. D. Goodman et al., "Predicting cirrhosis in patients with hepatitis $\mathrm{C}$ based on standard laboratory tests: results of the HALT-C cohort," Hepatology, vol. 42, no. 2, pp. 282-292, 2005.

[12] R. K. Sterling, E. Lissen, N. Clumeck et al., "Development of a simple noninvasive index to predict significant fibrosis in patients with HIV/HCV coinfection," Hepatology, vol. 43, no. 6, pp. 1317-1325, 2006.

[13] A. Vallet-Pichard, V. Mallet, B. Nalpas et al., "FIB-4: an inexpensive and accurate marker of fibrosis in HCV infection. Comparison with liver biopsy and fibrotest," Hepatology, vol. 46, no. 1, pp. 32-36, 2007.

[14] F. Ucar, S. Sezer, Z. Ginis et al., "APRI, the FIB-4 score, and Forn's index have noninvasive diagnostic value for liver fibrosis in patients with chronic hepatitis B," European Journal of Gastroenterology \& Hepatology, vol. 25, no. 9, pp. 1076-1081, 2013.

[15] J. Ma, Y. Jiang, and G. Gong, "Evaluation of seven noninvasive models in staging liver fibrosis in patients with chronic hepatitis B virus infection," European Journal of Gastroenterology \& Hepatology, vol. 25, no. 4, pp. 428-434, 2013.

[16] J. Cheng, J. Hou, H. Ding et al., "Validation of ten noninvasive diagnostic models for prediction of liver fibrosis in patients with chronic hepatitis B," PLoS One, vol. 10, no. 12, article e0144425, 2015.

[17] G. Xiao, J. Yang, and L. Yan, "Comparison of diagnostic accuracy of aspartate aminotransferase to platelet ratio index and fibrosis-4 index for detecting liver fibrosis in adult patients with chronic hepatitis B virus infection: a systemic review and meta-analysis," Hepatology, vol. 61, no. 1, pp. 292-302, 2015.

[18] Z. Zhang, G. Wang, K. Kang, G. Wu, and P. Wang, "The diagnostic accuracy and clinical utility of three noninvasive models for predicting liver fibrosis in patients with HBV infection," PLoS One, vol. 11, no. 4, article e0152757, 2016.

[19] F. M. Sanai, T. Farah, K. Albeladi et al., "Diminished accuracy of biomarkers of fibrosis in low replicative chronic hepatitis B," BMC Gastroenterology, vol. 17, no. 1, p. 101, 2017.

[20] Y. W. Tan, X. B. Zhou, Y. Ye, C. He, and G. H. Ge, "Diagnostic value of FIB-4, aspartate aminotransferase-to-platelet ratio index and liver stiffness measurement in hepatitis B virusinfected patients with persistently normal alanine aminotransferase," World Journal of Gastroenterology, vol. 23, no. 31, pp. 5746-5754, 2017.

[21] M. Dong, J. Wu, X. Yu et al., "Validation and comparison of seventeen noninvasive models for evaluating liver fibrosis in 
Chinese hepatitis B patients," Liver International, vol. 38, no. 9, pp. 1562-1570, 2018.

[22] M. Tag-Adeen, M. Z. Omar, F. M. Abd-Elsalam et al., “Assessment of liver fibrosis in Egyptian chronic hepatitis B patients: a comparative study including 5 noninvasive indexes," Medicine, vol. 97, no. 6, article e9781, 2018.

[23] M. Lemoine, Y. Shimakawa, S. Nayagam et al., "The gammaglutamyl transpeptidase to platelet ratio (GPR) predicts significant liver fibrosis and cirrhosis in patients with chronic HBV infection in West Africa," Gut, vol. 65, no. 8, pp. 1369-1376, 2016.

[24] D. P. Liu, W. Lu, Z. Q. Zhang et al., "Comparative evaluation of GPR versus APRI and FIB-4 in predicting different levels of liver fibrosis of chronic hepatitis B," Journal of Viral Hepatitis, vol. 25, no. 5, pp. 581-589, 2018.

[25] Y. C. Hu, H. Liu, X. Y. Liu et al., "Value of gamma-glutamyltranspeptidase-to-platelet ratio in diagnosis of hepatic fibrosis in patients with chronic hepatitis B," World Journal of Gastroenterology, vol. 23, no. 41, pp. 7425-7432, 2017.

[26] Q. Li, J. Song, Y. Huang et al., "The gamma-glutamyltranspeptidase to platelet ratio does not show advantages than APRI and fib- 4 in diagnosing significant fibrosis and cirrhosis in patients with chronic hepatitis B," Medicine, vol. 95, no. 16, article e3372, 2016.

[27] L. L. Schiavon, J. L. Narciso-Schiavon, M. L. G. Ferraz, A. E. B. Silva, and R. J. Carvalho-Filho, "The $\gamma$-glutamyl transpeptidase to platelet ratio (GPR) in HBV patients: just adding up?," Gut, vol. 66, no. 6, pp. 1169-1170, 2017.

[28] X. J. Lu, X. H. Li, Z. X. Yuan et al., "Assessment of liver fibrosis with the gamma-glutamyl transpeptidase to platelet ratio: a multicentre validation in patients with HBV infection," Gut, vol. 67, no. 10, pp. 1903-1904, 2018.

[29] Q. Li, C. Lu, W. Li, Y. Huang, and L. Chen, "Globulin-platelet model predicts significant fibrosis and cirrhosis in $\mathrm{CHB}$ patients with high HBV DNA and mildly elevated alanine transaminase levels," Clinical and Experimental Medicine, vol. 18, no. 1, pp. 71-78, 2018.

[30] Q. Li, L. Chen, and Y. Zhou, "Diagnostic accuracy of liver stiffness measurement in chronic hepatitis B patients with normal or mildly elevated alanine transaminase levels," Scientific Reports, vol. 8, no. 1, p. 5224, 2018.

[31] S. K. Sarin, M. Kumar, G. K. Lau et al., "Asian-Pacific clinical practice guidelines on the management of hepatitis B: a 2015 update," Hepatology International, vol. 10, no. 1, pp. 1-98, 2016.

[32] P. Bedossa and T. Poynard, "An algorithm for the grading of activity in chronic hepatitis C," Hepatology, vol. 24, no. 2, pp. 289-293, 1996.

[33] W. Rudin, Principles of Mathematical Analysis, McGraw-Hill, New York, 3rd edition, 1976.

[34] W. L. Winston and J. B. Goldberg, Operations Research: Applications and Algorithms, Brooks/Cole Publishing Company, Pacific Grove, CA, USA, 4th edition, 2003.

[35] T. Poynard, P. Halfon, L. Castera et al., "Standardization of ROC curve areas for diagnostic evaluation of liver fibrosis markers based on prevalences of fibrosis stages," Clinical Chemistry, vol. 53, no. 9, pp. 1615-1622, 2007.

[36] Q. Li, W. Li, Y. Huang, and L. Chen, "The gamma-glutamyl transpeptidase-to-platelet ratio predicts liver fibrosis and cirrhosis in HBeAg-positive chronic HBV infection patients with high HBV DNA and normal or mildly elevated alanine transaminase levels in China," Journal of Viral Hepatitis, vol. 23, no. 11, pp. 912-919, 2016.

[37] B. Liao, Z. Wang, S. Lin et al., "Significant fibrosis is not rare in Chinese chronic hepatitis B patients with persistent normal ALT," PLoS One, vol. 8, no. 10, article e78672, 2013.

[38] M. Kumar, S. K. Sarin, S. Hissar et al., "Virologic and histologic features of chronic hepatitis B virus-infected asymptomatic patients with persistently normal ALT," Gastroenterology, vol. 134, no. 5, pp. 1376-1384, 2008.

[39] Q. Xie, X. Hu, Y. Zhang, X. Jiang, X. Li, and J. Li, "Decreasing hepatitis $B$ viral load is associated with a risk of significant liver fibrosis in hepatitis B e antigen positive chronic hepatitis B," Journal of Medical Virology, vol. 86, no. 11, pp. 1828-1837, 2014.

[40] S. K. Goyal, A. K. Jain, V. K. Dixit et al., "HBsAg level as predictor of liver fibrosis in $\mathrm{HBeAg}$ positive patients with chronic hepatitis B virus infection," Journal of Clinical and Experimental Hepatology, vol. 5, no. 3, pp. 213-220, 2015.

[41] W.-K. Seto, C.-L. Lai, P. P. C. Ip et al., "A large population histology study showing the lack of association between ALT elevation and significant fibrosis in chronic hepatitis B," PLoS One, vol. 7, no. 2, article e32622, 2012.

[42] L. Xiao, J. Xian, Y. Li et al., "Parameters associated with significant liver histological changes in patients with chronic hepatitis B," ISRN Gastroenterology, vol. 2014, Article ID 913890, 6 pages, 2014.

[43] L. Feng, K. Sun, J. Zhang, G. Feng, and Y. Zhao, "A novel non-invasive index using AFP and APTT is associated with liver fibrosis in patients with chronic hepatitis B infection: a retrospective cohort study," BMJ Open, vol. 5, no. 9, article e008032, 2015.

[44] H. Dong, C. Xu, W. Zhou et al., "The combination of 5 serum markers compared to FibroScan to predict significant liver fibrosis in patients with chronic hepatitis B virus," Clinica Chimica Acta, vol. 483, pp. 145-150, 2018.

[45] Q. Han, S. Lou, Z. Liu et al., "Higher pretherapy and significant decrease during the first 12 months of therapy in serum laminin levels may associate with hepatitis B e antigen seroconversion in chronic hepatitis B patients treated with lamivudine," Clinical and Experimental Medicine, vol. 10, no. 4, pp. 245$251,2010$.

[46] Q. Li, C. Lu, W. Li, Y. Huang, and L. Chen, “The independent predictors of significant liver histological changes in chronic hepatitis B virus infection patients with persistently highnormal or low-normal alanine transaminase levels," Discovery Medicine, vol. 23, no. 124, pp. 19-25, 2017.

[47] G. L. Wong, V. W. Wong, P. C. Choi et al., "Clinical factors associated with liver stiffness in hepatitis $\mathrm{B}$ e antigen-positive chronic hepatitis B patients," Clinical Gastroenterology and Hepatology, vol. 7, no. 2, pp. 227-233, 2009.

[48] S. Alam, N. Ahmad, G. Mustafa, A. Shrestha, A. K. M. K. Alam, and M. Khan, "Evaluation of normal or minimally elevated alanine transaminase, age and DNA level in predicting liver histological changes in chronic hepatitis B," Liver International, vol. 31, no. 6, pp. 824-830, 2011.

[49] L. Wang, Z. Q. Zou, K. Wang, J. G. Yu, and X. Z. Liu, "Role of serum hepatitis B virus marker quantitation to differentiate natural history phases of HBV infection," Hepatology International, vol. 10, no. 1, pp. 133-138, 2016.

[50] L. Zhang, M. H. Li, W. H. Cao et al., "Negative correlation of serum hepatitis B surface antigen and hepatitis B e antigen 
levels with the severity of liver inflammation in treatmentnaïve patients with chronic hepatitis B virus infection," Chinese Medical Journal, vol. 130, no. 22, pp. 2697-2702, 2017.

[51] H. Wang, R. Yan, Y. Zhou, M. S. Wang, G. Q. Ruo, and M. J. Cheng, "Comparison of hepatitis B surface antigen and e antigen in predicting liver histology in hepatitis $\mathrm{B}$ e antigen-positive chronic hepatitis B patients," Hepatology International, vol. 8, no. 2, pp. 216-223, 2014.

[52] Y. Zan, Y. Zhang, and P. Tien, "Hepatitis B virus e antigen induces activation of rat hepatic stellate cells," Biochemical and Biophysical Research Communications, vol. 435, no. 3, pp. 391-396, 2013.

[53] J. Jia, J. Hou, H. Ding et al., “Transient elastography compared to serum markers to predict liver fibrosis in a cohort of Chinese patients with chronic hepatitis B," Journal of Gastroenterology and Hepatology, vol. 30, no. 4, pp. 756-762, 2015.

[54] S. Singh, A. J. Muir, D. T. Dieterich, and Y. T. Falck-Ytter, "American Gastroenterological Association Institute technical review on the role of elastography in chronic liver diseases," Gastroenterology, vol. 152, no. 6, pp. 1544-1577, 2017.

[55] C. Colletta, C. Smirne, C. Fabris et al., "Value of two noninvasive methods to detect progression of fibrosis among HCV carriers with normal aminotransferases," Hepatology, vol. 42, no. 4, pp. 838-845, 2005.

[56] J. Jia, J. Hou, H. Ding et al., "Prevalence and factors associated with failure of liver stiffness measurement using FibroScan in a prospective study of 2114 examinations," European Journal of Gastroenterology \& Hepatology, vol. 18, no. 4, pp. 411-412, 2006. 


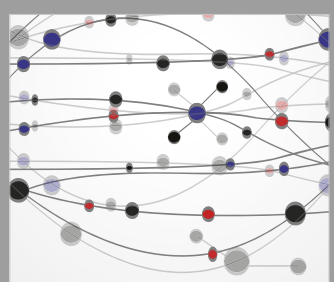

The Scientific World Journal
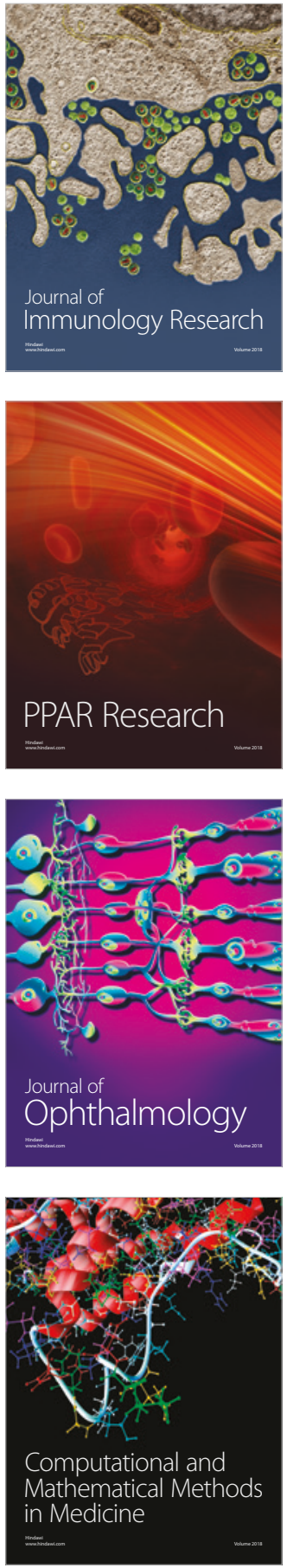

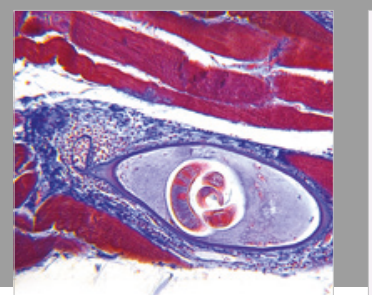

Gastroenterology Research and Practice

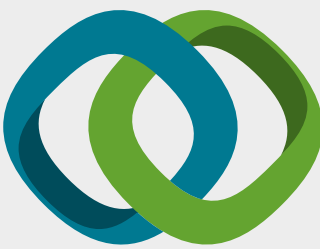

\section{Hindawi}

Submit your manuscripts at

www.hindawi.com
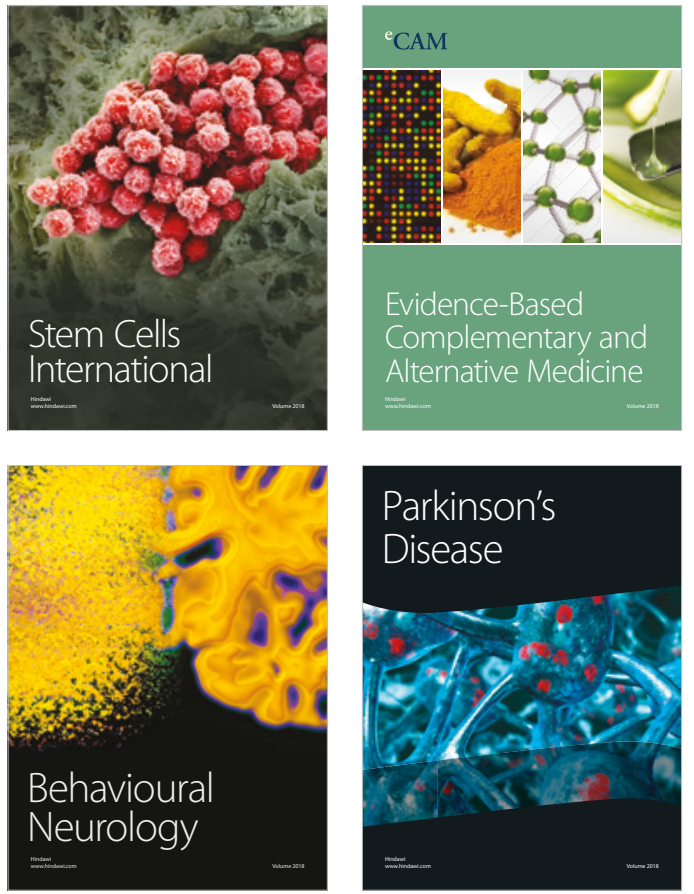

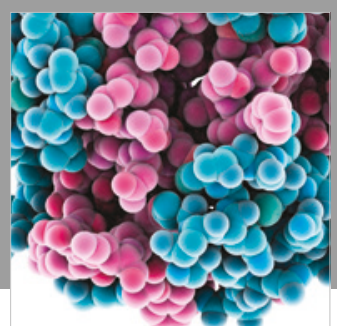

ournal of

Diabetes Research

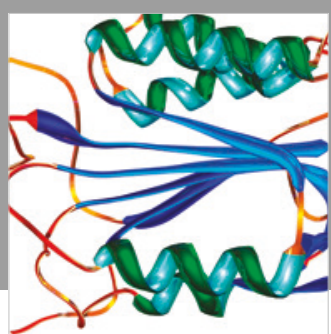

Disease Markers
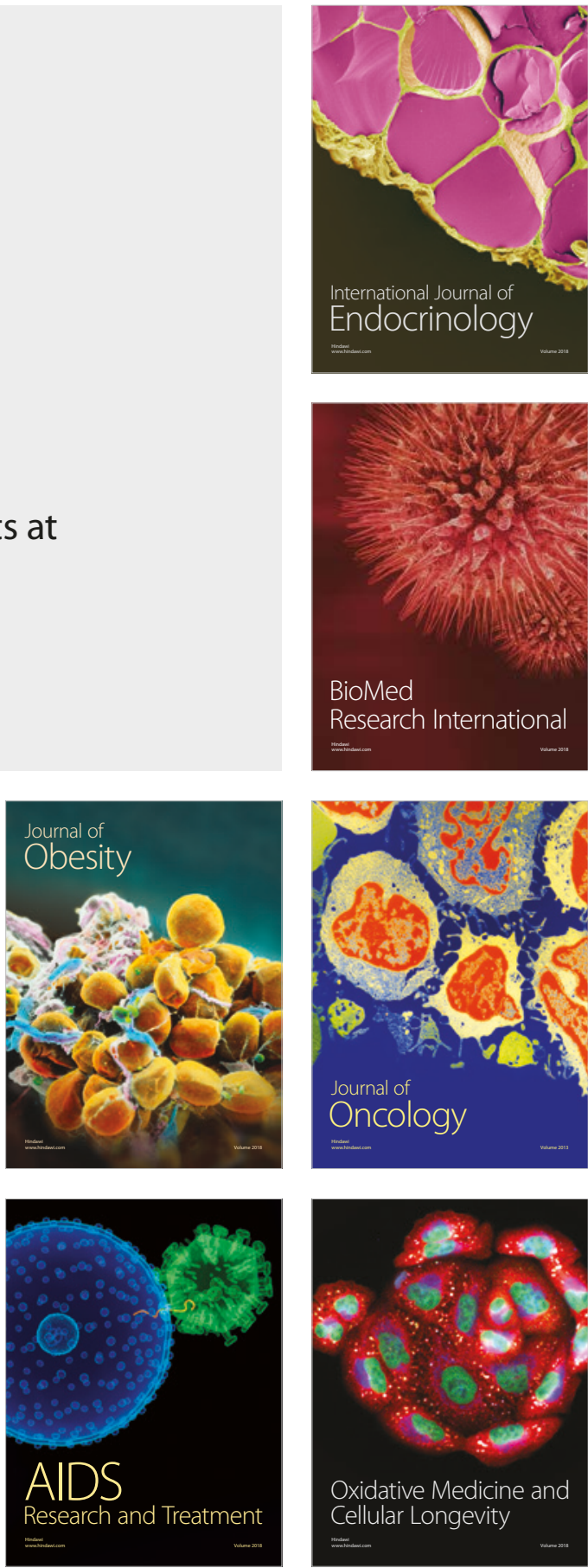